\title{
Vibration Control of Flexible Manipulator Using Filtered Inverse Controller
}

\author{
Waweru Njeri*, Minoru Sasaki and Kojiro Matsushita \\ Department of Mechanical Engineering, Gifu University, 1-1 Yanagido, Gifu, 501-1193, Japan \\ ${ }^{*}$ Corresponding author
}

\begin{abstract}
Our paper presents an inverse system based controller for the precise positioning of the manipulator end-effector. Lately, flexible manipulators are popular owing to their numerous advantages; light weight, low power consumption etc. However, due to their flexible nature, precise positioning of the end effector is still a serious challenge because of the accompanying link vibrations. This limits their applications to those fields where precise positioning or response time is not a factor. Inverse model was developed based on a mathematical model developed, linearized in Maple/Maplesim and exported to MATLAB. Practical experiments were carried out in dSPACE environment. Results show that precise positioning with minimal vibration can be achieved using this technique.
\end{abstract}

Keywords-inverse system; internal dynamics; vibration

\section{INTRODUCTION}

The need of multilink manipulator is due to advantages such as; high speed, cheap due to less materials requirements than their rigid counterparts, light in weight which in turn calls for small actuators, energy efficiency, mobility, swift and high payload to weight ratio[1],[2]. Flexible manipulators find applications in areas like soldering of computer motherboard, precision welding and positioning of the read-write head of a hard drive to mention just a few. For a flexible manipulator, this is even more difficult owing to the flexible nature of the links. At high operational speed, the inertial forces increases leading to vibration of the links. This brings about delays in the precise positioning of the end-effecter. Also, being highly coupled, link vibrations become more severe with additional links. A lot of research has been done on this front by the use of methods such as the application of fixed digital filters. Also, adaptive filters to filter out vibration modes have been proposed[3]. In the same respect, Proportional Integral Differential(PID) controllers have been employed to mitigate these notorious problems[4].

In theory, there are two types of inversion: right and left inversion as shown in Figure 1. A left inversion will yield the input signal to the plant when excited from the knowledge of its output signal as shown in Figure 1a whereas in right inversion, yields the necessary input to the plant for the desired plant output as shown in Figure 1b[5]. General applications of Left and right inversion are fault detection and the feed forward control respectively. The research reported in this paper is based on the right inversion.

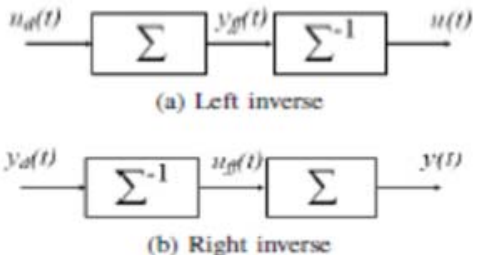

FIGURE I. FINVERSE CONTROLLER CONFIGURATION

Consider a generic dynamical system $\Sigma$ where $\mathrm{u}(\mathrm{t})$ and $\mathrm{y}(\mathrm{t})$ respectively represent the inputs and outputs. The Model inversion is a procedure to find the feedforward input function $u_{f f}$ to yield the given desired output function $y_{d}(t)$ of the system under consideration. In other words, it is like to build an inverse of $\Sigma$ where the input corresponds to the original output and vice versa [6] - [9].

Inversion of dynamic system is not a new idea but has been there since early sixties first formulated by Brocket and Mesarovic in 1965[10]. Silverman[7] later developed an iterative inversion schemes by successive differentiation and partitioning the output variable. Massey and Sain in the same period developed a different scheme from that of Silverman[8]. In the decade that followed, Moylan [5] refined the previous work and developed another algorithm. Hirschorn extended the procedures earlier developed for linear system inversion to nonlinear systems.

Key concerns in the development of an inverse model are the existence of the inverse model and the stability of the resulting inverse model. Given a linear time-invariant(LTI) single-input single-output (SISO) system expressed by the transfer function representation, the inversion procedure is equivalent to exchanging between the numerator and the denominator. The resulting transfer function is stable if the zeros of the system are stable. In other words, the inverse is stable if the zeros of the system under consideration are on the open left hand side of the s-plane, i.e. the system is minimum phase plant, otherwise if the system is non-minimum phase it results in an unstable inverse.

Classical techniques however were limited to minimum phase systems. For non-minimum phase systems, the yielded inverse is unstable. Devasia[11], an author who has done. remarkable research in inversion theory, especially for nonminimum phase systems, successfully managed to invert a non- minimum phase system by isolating the internal dynamic and decomposing them into stable and unstable dynamics. The stable dynamics are solved by integrating forward in time 
where the unstable one solved to preview technique. Detailed mathematical presentation of the preview based technique can be found in [12]-[14]. For non-hyperbolic systems, devasia proposed a solution in [12]. However, this is only applicable to systems which are completely controllable.

To this end, we developed an inverse controller inherent to the work by devasia. Pole placement technique was used to stabilize the internal dynamics of the non-hyperbolic and uncontrollable plant. Having being augmented with lowpass filters, the inverse model is used as a feedforwad controller to a two link 3D flexible manipulator. Results presented in section $\mathrm{V}$ shows significant reduction in the link strain with the employment of the inverse controller.

\section{Model Formulation ANd VAlidation}

The plant is three dimensional two link flexible manipulator structured as in Figure 2. It has three rotary joints driven by de servo motors and two flexible links $l_{1}$ and $l_{2}$. The control system consists of a computer, AC and DA converters all managed by dSPACE and Matlab softwares. Measure of angular position, velocity is achieved by encoders places in the servomotors while and link strain measurement is done by strain gauges positioned at the bottom of each link. The manipulator was modelled and linearized in Maple/Maplesim. A mathematical model of the plant was validated with the performance of the actual plant. Then, the inverse model was developed based on this minimal state space representation of the manipulator.

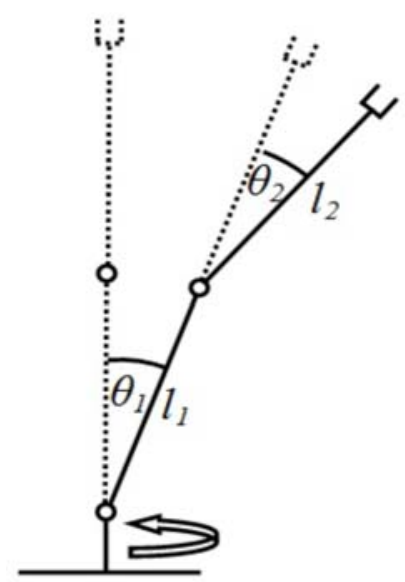

FIGURE II. MANIPULATOR MODEL

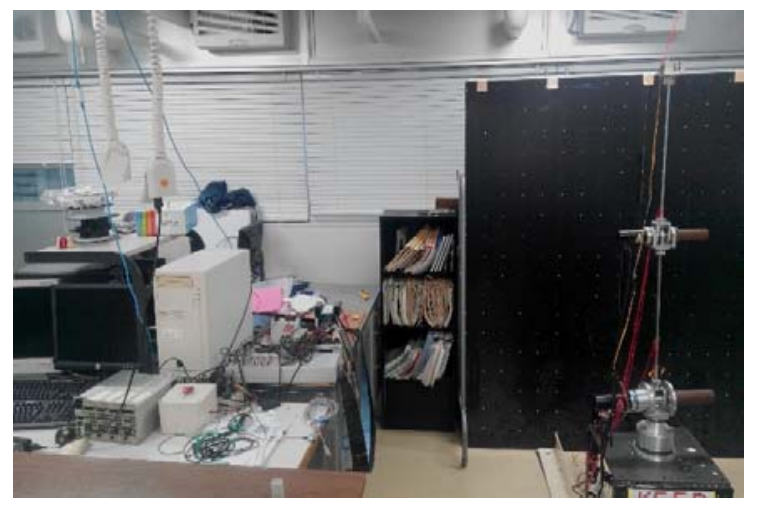

FIGURE III. EXPERIMENTAL SETUP

\section{DeVElopMent of The INVERSE System}

Consider a LTI continuous time square system $\Sigma$, let the triplet $\mathrm{A}, \mathrm{B}$ and $\mathrm{C}$ be a minimal state-space representation. It is assumed that the system is stable or stabilized by negative

Feedback

$$
\begin{gathered}
\dot{x}(\mathrm{t})=\mathrm{Ax}(\mathrm{t})+\mathrm{Bu}(\mathrm{t}) \\
\mathrm{y}(\mathrm{t})=\mathrm{Cx}(\mathrm{t})
\end{gathered}
$$

Where $\mathrm{x}(\mathrm{t}) \in R^{n}, \mathrm{u}(\mathrm{t}) \in R^{p}, y=\left(y_{1}, y_{2}, \cdots, y_{p}\right)^{T} \in R^{p}, A \in$ $R^{n \times n}, B \in R^{n \times p}$ and $C \in R^{p \times n}$. If $C_{\mathrm{i}}$ denotes the $\mathrm{i}_{\text {th }}$ row of the output matrix $C$, the system is said to have a well-defined relative degree $r \triangleq\left(r_{1}, r_{2}, \cdots r_{p}\right)^{T}$ if $C_{i} A^{l} B=0, \forall l<r_{i}-$ $1,1 \leq i \leq p[15]$.

Differentiating $i_{t h}$ output $r_{i}$ times in time yields

$$
y^{\left(r_{k}\right)}=C_{k} A^{\left(r_{k}\right)} x+C_{k} A^{\left(r_{k}-1\right)} B u
$$

where $C_{k}$ is the $k_{t h}$ row of the output matrix $C$ and $1<\mathrm{k}<\mathrm{p}$. In vector form

$$
y^{(r)}(\mathrm{t})=\mathrm{A}_{x} \mathrm{x}(\mathrm{t})+B_{y} \mathrm{u}(\mathrm{t})
$$

where

$$
\begin{aligned}
y^{(r)} & \triangleq\left[y_{1}^{\left(r_{1}\right)}, y_{2}^{\left(r_{2}\right)}, \cdots, y_{p}^{\left(r_{p}\right)}\right]^{T} \\
B_{y} & \triangleq\left[C_{1} A^{\left(r_{1}\right)}, C_{1} A^{\left(r_{2}\right)}, \cdots, C_{1} A^{\left(r_{p}\right)}\right]^{T} \\
A_{x} & \triangleq\left[C_{1} A^{\left(r_{1}-1\right)}, C_{1} A^{\left(r_{2}-1\right)}, \cdots, C_{1} A^{\left(r_{p}-1\right)}\right]^{T}
\end{aligned}
$$

By is invertible because of the well-defined relative degree assumption. From equation (3), we can see that the control law is

$$
u_{f f} \quad B_{y}^{-1}\left[y_{d}^{(r)}-A_{x} x(t)\right] \quad \forall t \in(-\infty, \infty)
$$

Then, there exist a state transformation $\mathrm{T}$

$$
[\xi(t) \eta(t)]=T x(t)
$$

which transforms the states to internal dynamics $\eta(t)$ and the output and its derivatives in time up to (r1-1) as 


$$
\left[y_{1}, \cdots, y_{1}^{\left(r_{1}-1\right)}, \cdots, y_{p}, \cdots, y_{p}^{\left(r_{1}-1\right)}\right]
$$

The expression of the new system after coordinate transformation is

$$
\begin{array}{ll}
\dot{\xi} & \hat{A}_{1} \xi+\hat{A}_{2} \eta+\hat{B}_{1} u \\
\dot{\eta} & \hat{A}_{3} \xi+\hat{A}_{4} \eta+\hat{B}_{3} u
\end{array}
$$

where

$$
\hat{A}=\left[\begin{array}{ll}
\hat{A}_{1} & \hat{A}_{2} \\
\hat{A}_{3} & \hat{A}_{4}
\end{array}\right]=T^{-1} A T \text { and } \hat{B}=\left[\begin{array}{l}
\hat{B}_{1} \\
\hat{B}_{3}
\end{array}\right]
$$

The control law to maintain the exact tracking can be written

As

where

$$
u_{f f}=B_{y}^{-1}\left[y_{d}^{r}-A_{\xi} \xi(t)-A_{\eta} \eta(t)\right]
$$

Also the internal dynamics

$$
\begin{aligned}
\dot{\eta} & \hat{A}_{3} \xi+\hat{A}_{4} \eta+\hat{B}_{2} B_{y}^{-1}\left[y_{d}^{r}-A_{\xi} \xi(t)-A_{\eta} \eta(t)\right] \\
& \hat{A}_{\eta} \eta(t)+\hat{B}_{\eta} Y
\end{aligned}
$$

Where

$$
\begin{array}{ll}
\hat{A}_{\eta} & \hat{A}_{4}-\hat{B}_{2} B_{y}^{-1} A_{\eta} \\
\hat{B}_{\eta} & \left.\hat{A}_{3}-\hat{B}_{2} B_{y}^{-1} A_{\xi}+\hat{B}_{2} B_{y}^{-1}\right] \\
\vdots & {\left[\begin{array}{ll}
\xi & y_{d}^{(r)}
\end{array}\right]^{T}}
\end{array}
$$

in the same respect, equation 3 can now be written as

$$
\begin{gathered}
u_{f f} \quad B_{y}^{-1}\left[y_{d}^{(d)}-A_{\xi} \xi(t)-A_{\eta} \eta(t)\right] \\
-B_{y}^{-1} A_{\eta} \eta(t)-\left[B_{y}^{-1} A_{\xi}-B_{y}^{-1}\right] Y \\
C_{\eta} \eta(t)+D_{Y} Y(t)
\end{gathered}
$$

Where

$$
\begin{array}{cc}
C_{\eta} & -B_{y}^{-1} A_{\eta} \\
D_{Y} & -\left[B_{y}^{-1} A_{\xi}-B_{y}^{-1}\right]
\end{array}
$$

equation (7) together with equation (8) form the inverse system and can be represented in state space form as in Figure 4

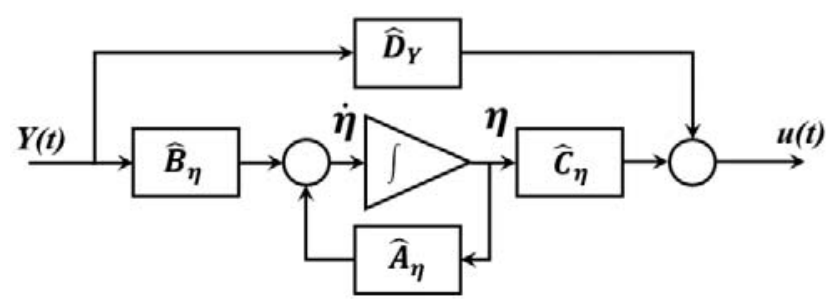

FIGURE IV. BLOCK DIAGRAM OF THE INVERSE SYSTEM

IV. INVERTING the FleXible Manipulator Model

The model has 17 states as follows:
- $x_{1}(t)=i_{1}(t)$
- $x_{2}(t)=w_{1}(t)$
- $x_{10}(t)=\theta_{1}(t)$
- $x_{3}(t)=\dot{w}_{1}(t)$
- $x_{11}(t)=\dot{\theta}_{1}(t)$
- $x_{4}(t)=w_{2}(t)$
- $x_{12}(t)=\theta_{2}(t)$
- $x_{5}(t)=\dot{w}_{2}(t)$
- $x_{13}(t)=\theta_{2}(t)$
- $x_{6}(t)=w_{3}(t)$
- $x_{7}(t)=\dot{w}_{3}(t)$
- $x_{8}(t)=w_{4}(t)$
- $x_{14}(t)=\theta_{3}(t)$
- $x_{15}(t)=\dot{\theta}_{3}(t)$
- $x_{16}(t)=i_{3}(t)$
- $x_{9}(t)=\dot{w}_{4}(t)$
- $x_{17}(t)=i_{2}(t)$

where $i_{j}$ denotes joint $j$ motor current, $\theta_{j}, \dot{\theta}_{j}$ angle and velocity of joint $\mathrm{j}=1,2,3$ respectively whereas $\left(w_{1} ; w_{2}\right),\left(w_{3} ; w_{4}\right)$ and their derivatives denotes the flexure variable for links 1 and 2 respectively.

Having three inputs, three outputs and a relative degree of three $(r=3)$, the internal dynamics were taken as the flexure variables:

$$
\eta(t)=\left[\begin{array}{l}
x_{2}(t) \\
x_{3}(t) \\
x_{4}(t) \\
x_{5}(t) \\
x_{6}(t) \\
x_{7}(t) \\
x_{8}(t) \\
x_{9}(t)
\end{array}\right]
$$

whereas the output and its derivatives were takes as the three joint angles and their derivatives. Figure 5 below show the pole zero map of the plant and the inverse system. 


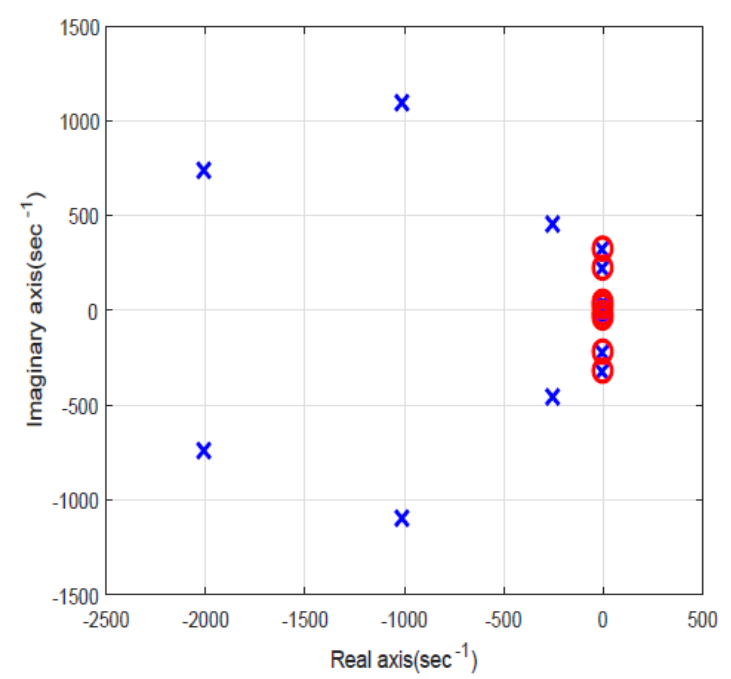

(a) Pole-zero map of the plant

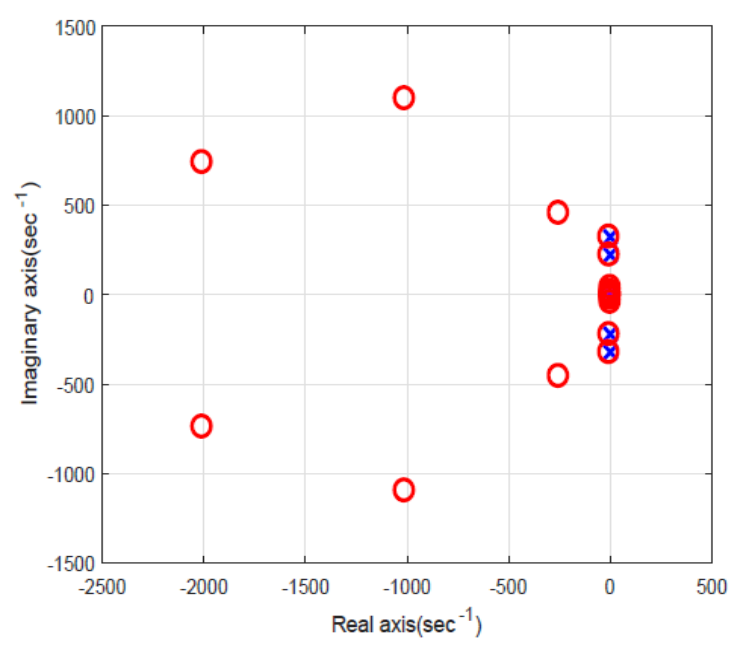

(b). Pole-zero map of the inverse

FIGURE V. POLES AND ZEROS OF THE PLANT AND ITS INVERSE

The system was found to be neither state controllable nor observable. Also, from the figure, we can see that the system is non-hyperbolic i.e., we have poles on the imaginary axis. This has the negative implication that the poles of the system cannot be arbitrarily placed. However, it was found that after transformation, $(A \eta ; B \eta)$ is fully controllable and the poles in the subsystem involved with the internal dynamics (flexure variables) can be arbitrarily place.

Non-hyperbolicity is a situation where the poles of a dynamic system are located on the imaginary axis. Whereas the system is not unstable, this scenario is undesirable especially in vibration control as the internal dynamics, flexure variables in this case remains constant with time. To make the system hyperbolic, pole assignment method was employed in the internal dynamic systems $(A \eta ; B \eta)$. With all the poles arbitrarily placed on the left hand side of the s-plane, the convergence of the internal dynamics is assured. The internal dynamics $\eta(t)$ can be solved conventionally by integrating forward in time as

$$
\eta(t)=\int_{-\infty}^{t} e^{A_{\eta}(t-\tau)} B_{\eta} Y_{d}(\tau) d \tau
$$

Since the system involves the derivatives of the desired output, it is required that this signal be differentiable up to degree $r^{p}$. This was solved by augmenting the inverse system with lowpass filter as in the internal model architecture[16], [17] of the form

$$
f(s)=\frac{1}{(\lambda s+1)^{n}}
$$

The order of the filter $n$ is chosen large enough to make the inverse system proper. On the other hand, the adjustable parameter $\lambda$ determine the filter rolloff which in turn determines the speed of response. Increasing the values of $\lambda$ makes the response sluggish and vice versa.

\section{EXPERIMENTAL RESULTS AND DISCUSSION}

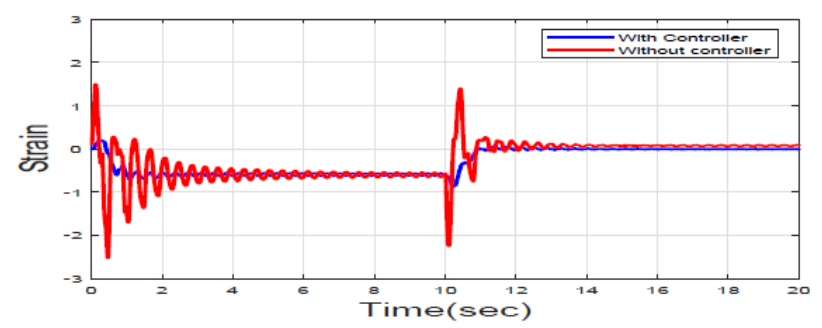

(a). $\lambda=0.3$

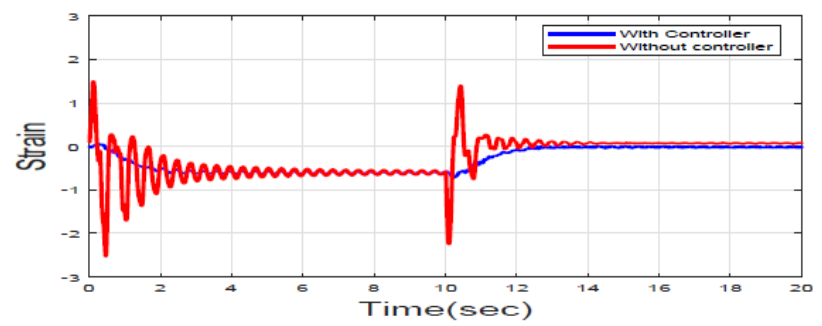

(b). $\lambda=0.5$

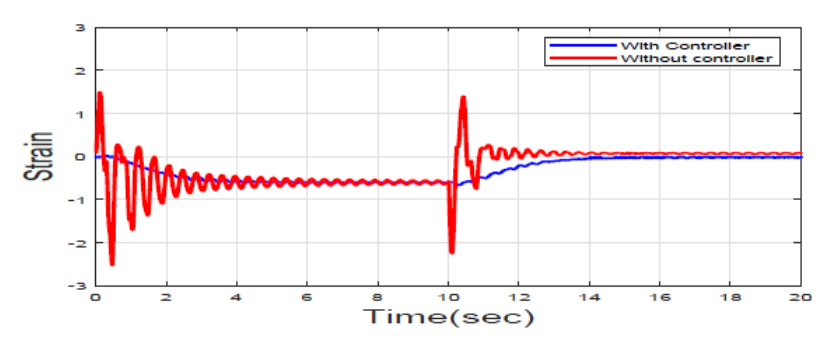

(c). $\lambda=0.7$

FIGURE VI. STRAIN, LINK 1 IN PLANE

Figure 6, 7 and 8 shows the in-plane, torsional and out of plane strain for link 1 and link 2 for values of $\lambda=0.3,0.5$ and 0.7 respectively. There was a significant reduction in link vibration with the introduction of the inverse controller. This is attributed to the fact that the internal dynamics corresponding to the vibrations are suppressed in the inverse system and any residue is further filtered out by the augmenting low pass filters. 


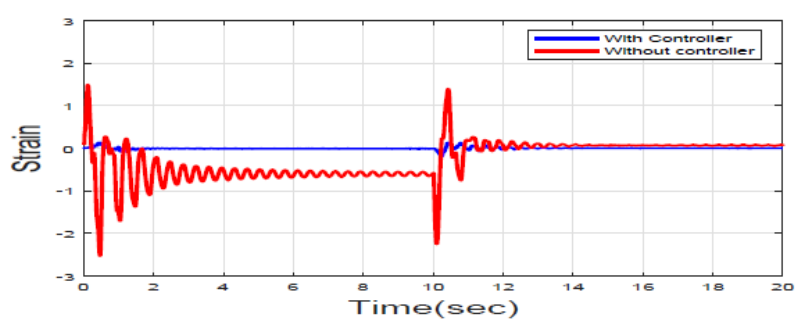

(a). $\lambda=0.3$

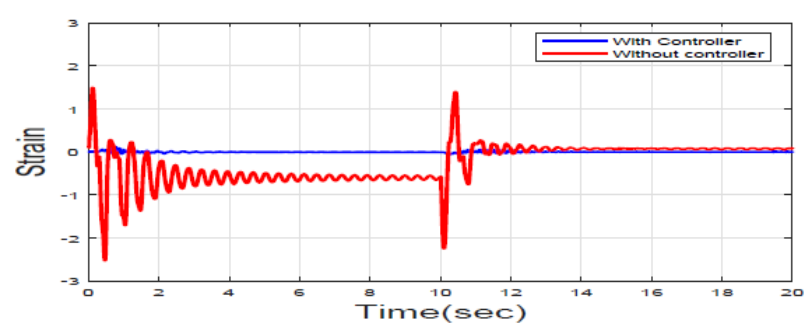

(a). $\lambda=0.3$

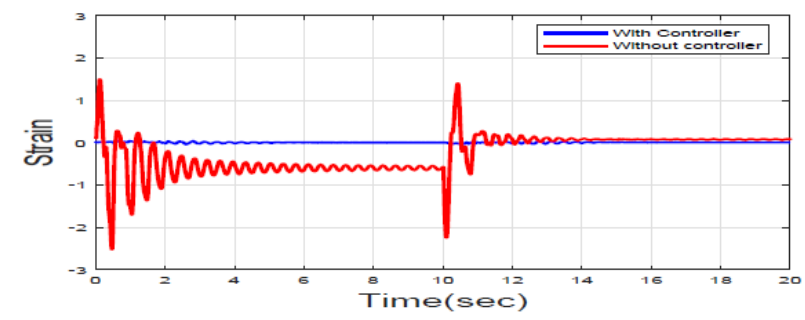

(a). $\lambda=0.3$

FIGURE VII. TORSIONAL STRAIN

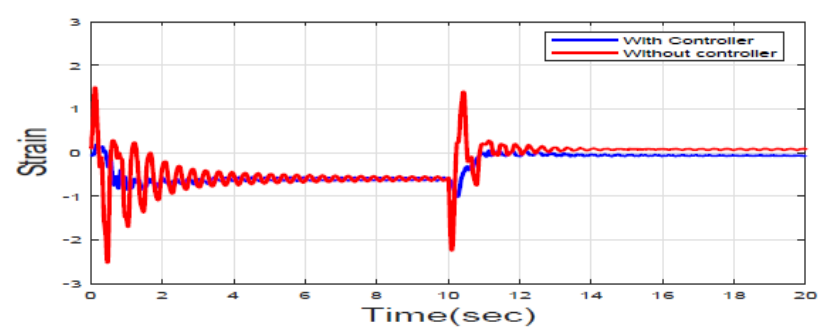

(a). $\lambda=0.3$

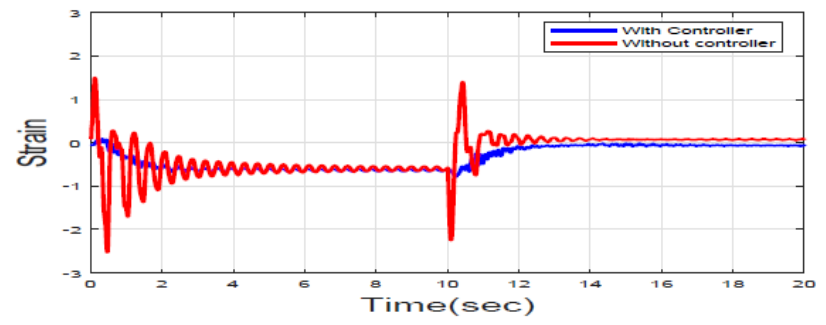

(a). $\lambda=0.5$

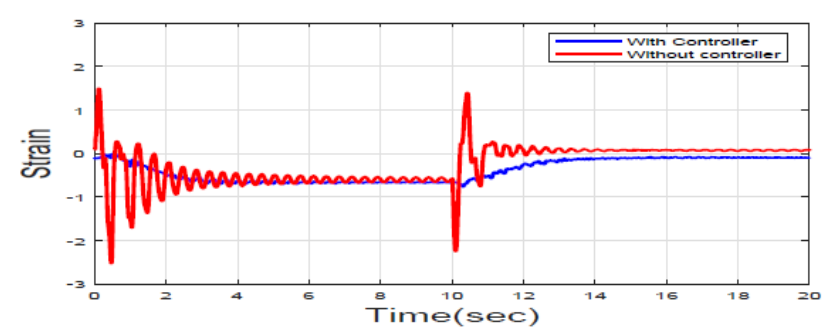

(a). $\lambda=0.7$

FIGURE VIII. STRAIN, LINK 2 OUT OF PLANE

We can also see that an increase in the value of $\lambda$ is accompanied by sluggish response as well as more reduction in link vibration. A closer look at the first 10 seconds of the strain information, the response with the controller takes 2 seconds for $\lambda=0.3,3$ seconds for $\lambda=0.5$ and 4 seconds for $\lambda=0.7$ which is very short in comparison to the response without the controller, which doesn't settle to the desired position for the entire period. In the last 10 seconds, though the settling time is 4 seconds, the response with the inverse controller is way superior.

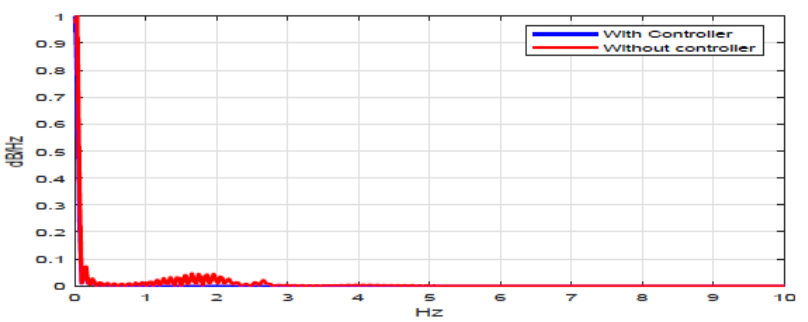

(a). $\lambda=0.3$

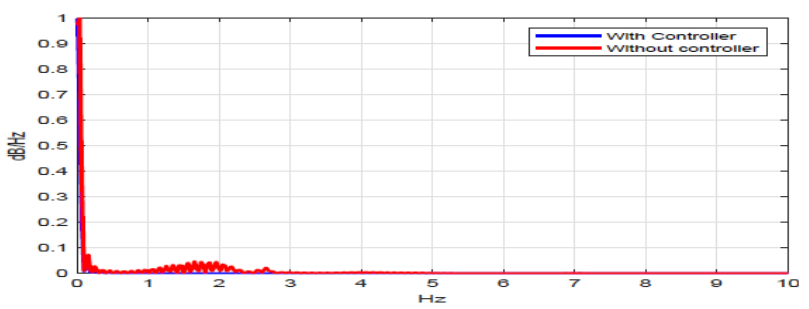

(a). $\lambda=0.5$

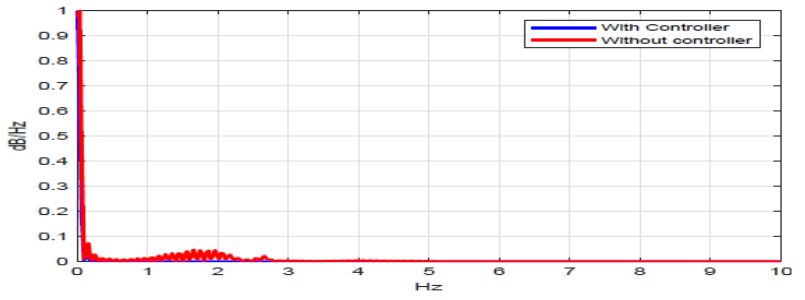

(a). $\lambda=0.7$

FIGURE IX. STRAIN SPECTRAL POWER DENSITY, LINK 1 IN PLANE 


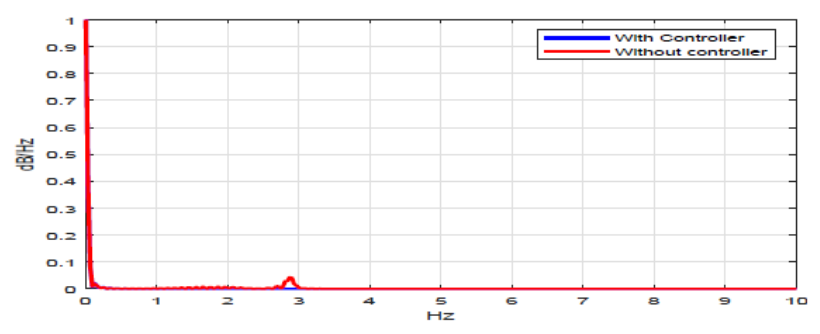

(a). $\lambda=0.3$

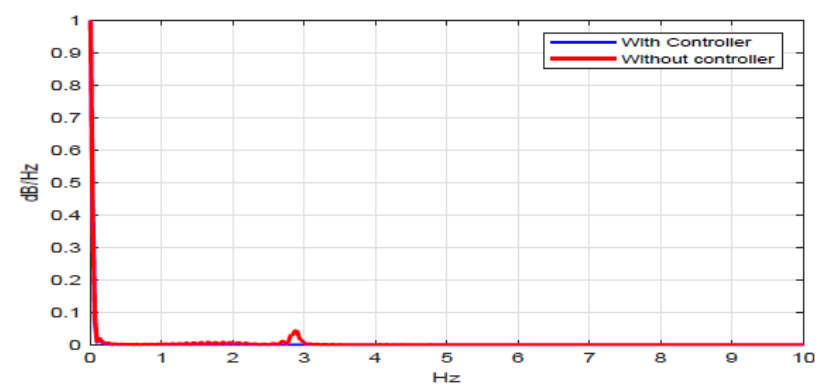

(a). $\lambda=0.5$

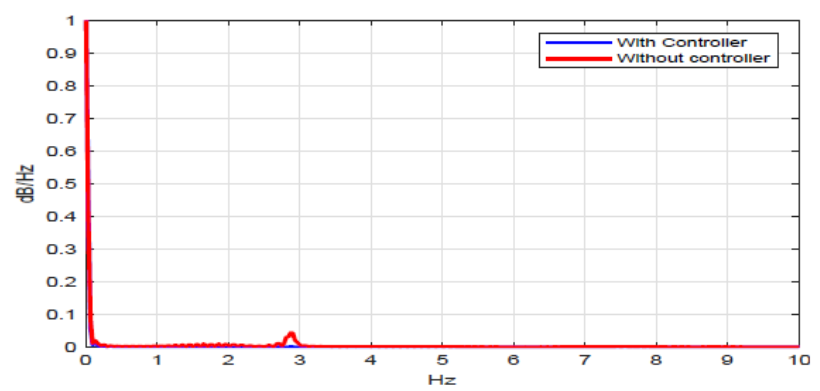

(a). $\lambda=0.7$

FIGURE X. STRAIN SPECTRAL POWER DENSITY, TORSION

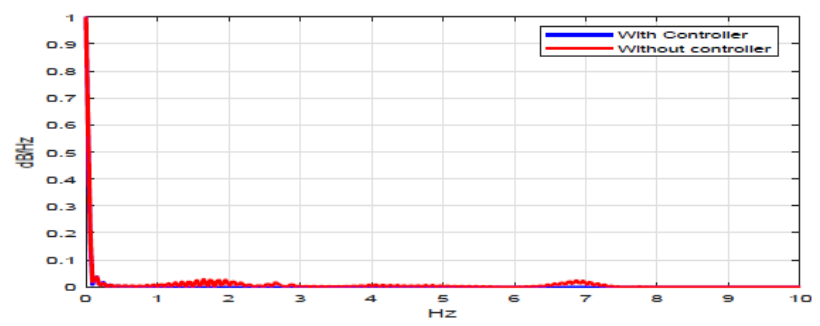

(a). $\lambda=0.3$

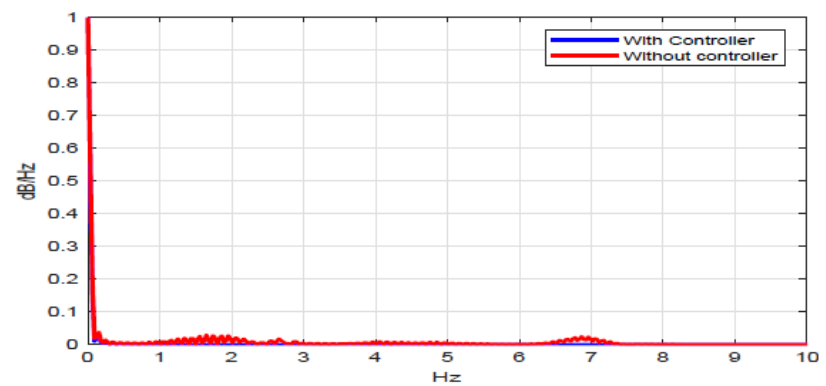

(a). $\lambda=0.5$

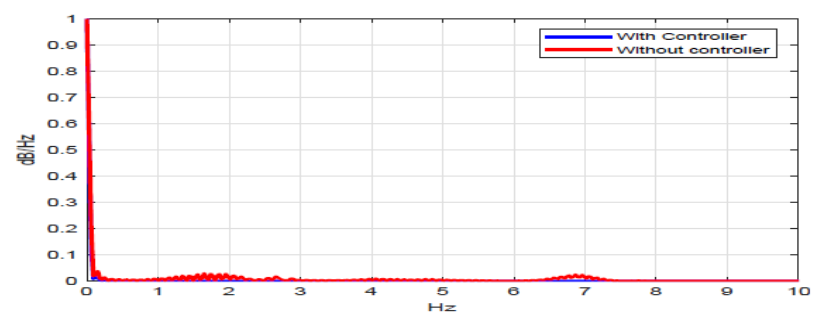

(a). $\lambda=0.7$

FIGURE XI. STRAIN SPECTRAL POWER DENSITY, LINK 2 IN PLANE

Figure 9, 10 and 11 shows the in-plane, torsional and outof-plane strain spectral power densities for link 1 and link 2 . Peaks between $3 \mathrm{~Hz}$ and $7 \mathrm{~Hz}$ correspond the link vibration dominant modes. This confirms the improvement introduced by the inverse controller. Figure 12 shows the variation of the strain spectral power density with different values of $\lambda$.

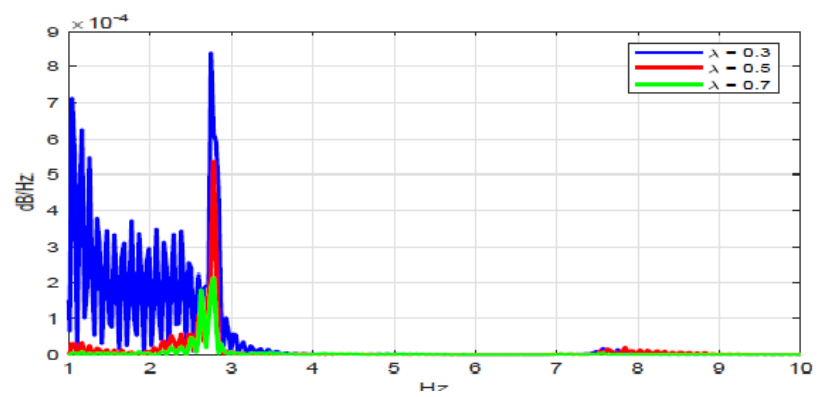

(a) Strain spectral power density, link 1 in plane

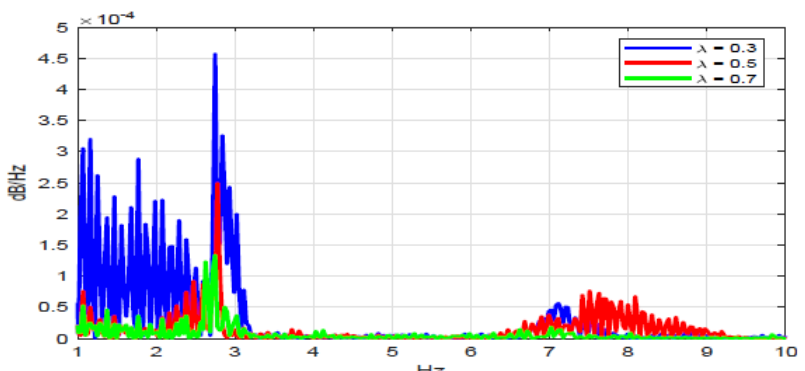

(b) Strain spectral power density, link 1 torsion

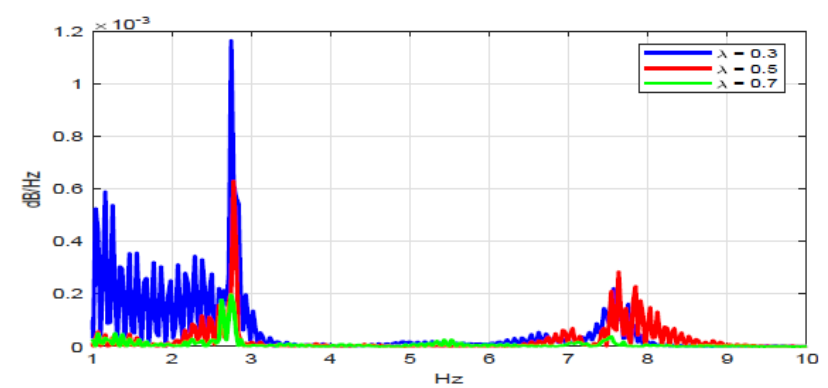

(c) Strain spectral power density, link 2 in plane

FIGURE XII. VARIATION OF STRAIN SPECTRAL POWER DENSITY WITH $\lambda$

Significant reduction in the vibration modes is evident at $3 \mathrm{~Hz}$ and minimal reduction for the $7 \mathrm{~Hz}$ modes. 


\section{CONCLUSION}

In this paper, an inverse system for a multilink flexible manipulator is successfully developed and used as a controller. The results presented shows that link vibrations are significantly reduced in comparison to a system without inverse controller.

\section{REFERENCES}

[1] A. A. Ata, W. F. Fares, and M. Y. Sa'adeh, "Dynamic analysis of a twolink flexible manipulator subject to different sets of conditions," Procedia Engineering, International Symposium on Robotics and Intelligent Sensors 2012, vol. 41, p. 1253 1260, 2012. www.sciencedirect.com.

[2] K. Lochan, B. K. Roy, and B. Subudhi, "A review on two-link flexible manipulators," Science irect, Annual reviews in control, vol. 42, pp. 346-367, 2016.

[3] Z. Chu and J. Cui, "Vibration control of maneuvering spacecraft with flexible manipulator using adaptive disturbance rejection filter and command shaping technology," in 2012 Sixth International Conference on Internet Computing for Science and Engineering, pp. 97-101, April 2012.

[4] T. A. Rahman, A. Asarry, and N. A. A. Jalil, "Active vibration control of a flexible beam structure using chaotic fractal search algorithm," Procedia Engineering, vol. 170, pp. 299 - 306, 2017. Engineering Physics International Conference 2016 fEPICg 2016.

[5] P.J. Moylan, "Stable inversion of linear systems," IEEE Transactions on Automatic Control, pp. 74-78, 1977.

[6] J. Chen and T. Huang, "Applying neural networks to on-line updated pid control for nonlinear process control," Journal of Process Control, vol. 14, pp. 211-230, 2004. www.elsevier.com/locate/jprocont.

[7] L. M. Silverman, "Inversion of multivariable linear systems," IEEE Transactions on Automatic Control, vol. 14, pp. 270-276, 1969.

[8] M. K. Sain and J. L. Massey, "Invertibility of linear time-invariant dynamical systems," IEEE Transactions on Automatic Control, vol. 14, pp. 141-149, 1969.

[9] Z. Xin-Jian and T. Li, "A new method for contructing the inversion of multivariable linear systems," Chinese Quarterly Journal of Mathematics, vol. 16, pp. 18-24, 2001.

[10] R. Hirschorn, "Invertibility of multivariable nonlinear control systems," IEEE Transactions on Automatic Control, vol. 24, pp. 855-865, December 1979.

[11] S. Devasia, D. Chen, and B. Paden, "Nonlinear inversion-based output tracking," IEEE Transactions on Automatic Control, vol. 41, pp. 930942, Jul 1996.

[12] S. Devasia, "Output tracking with Nonhyperbolic and Near Nonhyperbolic Internal Dynamics: Helicopter Hover Control,” Journal of Guidance, Control and Dynamics, vol. 20, pp. 573-580, May-June 1997.

[13] Qingze Zou and Santosh Devasia, "Preview-Based Stable-Inversion for Output Tracking of Linear Systems," Journal of Dynamic Systems, Measurement, and Control, vol. 121, pp. 625-630, 1999.

[14] Q. Zou, "Optimal preview-based stable-inversion for output tracking of nonminimum-phase linear systems," Automatica, vol. 45, no. 1, pp. 230 $-237,2009$.

[15] D. Liberzon, A. S. Morse, and E. D. Sontag, "Output-input stability and minimum-phase nonlinear systems," IEEE Transactions on Automatic Control, vol. 47, pp. 422-436, Mar 2002.

[16] M. Morari and E. Zafiriou, "Roubust process control. englewood cliffs," 1989.

[17] D. E. Rivera, "Internal model control: A comprehensive view," Arizona State University, Tempe, Arizona, pp. 85287-6006, 1999. 